\title{
ДОПУСТИМЫЕ И МИНИМАКСНАЯ БАИЕСОВСКИЕ ОЩЕНКИ БИНОМИАЛЬНОЙ ВЕРОЯТНОСТИ ПРИ ОГРАНИЧЕНИЯХ НА АПРИОРНОЕ РАСПРЕДЕЛЕНИЕ
}

\author{
(Представил Н. Алумяэ)
}

Рассмотрим задачу байесовского оценивания вероятности $p$ по $n$ независимым наблюдениям $x_{1}, x_{2}, \ldots, x_{n}$ над биномиальной случайной величиной $X$, т. е. $P(X=1)=p, P(X=0)=1-p$, при условии, что априорное распределение параметра $p$ принадлежит фиксированному классу распределений. Аналогичная задача рассматривалась в $\left[{ }^{1,2}\right]$, где класс априорных распределений определялся ограничениями на моменты этих распределений, в то время как в настоящей статье эти ограничения накладываются на форму распределений.

Сформулируем рассматриваемую задачу. Пусть $f\left(x_{1}, x_{2}, \ldots, x_{n}\right)$ оценка случайного, независимого от $X$ параметра $p$. Для квадратичной потери байесовский риск $r(f, F)$ имеет вид

$$
r(f, F)=\int_{0}^{1} E_{p}\left(f\left(x_{1}, x_{2}, \ldots, x_{n}\right)-p\right)^{2} d F(p)
$$

где $F(p)$ - априорное распределение параметра $p$, про которое известно лишь то, что оно принадлежит заданному классу $\mathcal{F}$.

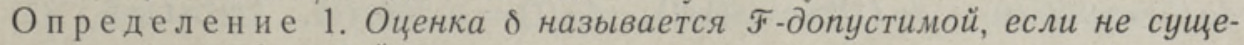
ствует оценки $\delta_{0}$ такой, что

$$
r\left(\delta_{0}, G\right) \leqslant r(\delta, G) \quad \forall G \in \mathcal{F}
$$

$u$

$$
r\left(\delta_{0}, G\right)<r(\delta, G)
$$

хотя бы для одного $G \in \mathcal{T}$.

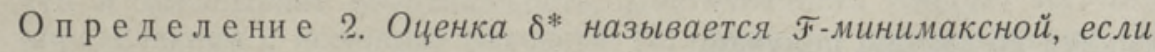

$$
\inf _{\delta} \sup _{G \in F} r(\delta, G)=\sup _{G \in F} r\left(\delta^{*}, G\right) \text {. }
$$

В настоящей статье решается задача описания $\mathcal{F}$-допустимых оценок и $\mathcal{F}$-минимаксной оценки в случае, когда $\mathcal{F}$ является классом распределений $G$ таких, что

1) $G(p+0)+G(1-p)=1,0 \leqslant p \leqslant 1$,

2) $G(p)$ вогнута на интервале $(0,1 / 2)$.

Обозначим через $B(\mathcal{T})$ класс байесовских оценок параметра относительно распределений из $\mathcal{F}$, т. е.

$$
B(\mathcal{F})=\left\{\bar{\delta} / \exists F \in \mathcal{F}: \inf _{\delta} r(\delta, F)=r(\bar{\delta}, F)\right\}
$$


Т е о р ем а. Класс $\mathcal{F}$-допустимых оценок совпадает с $B$ (F)). Доказ ательство. Рассмотрим семейство функций распределения $P_{t}(p), 0 \leqslant t \leqslant 1 / 2$, определенных следующим образом:

$P_{t}(p)=\left\{\begin{array}{l}0, p \leqslant 0 \\ \frac{p}{2 t}, \quad 0<p \leqslant t \\ 1 / 2, \quad t<p \leqslant 1-t \\ \frac{p}{2 t}+\left(1-\frac{1}{2 t}\right), \quad 1-\mathrm{t}<p \leqslant 1 \\ 1, p>1\end{array}\right.$

$$
P_{0}(p)= \begin{cases}0, & p \leqslant 0 \\ 1 / 2, & 0<p \leqslant 1 \\ 1, & p>1\end{cases}
$$

Очевидно, что $P_{t}(p) \in \mathscr{F}, 0 \leqslant t \leqslant 1 / 2$.

Лемм а 1. Для того, чтобы $G \in \mathcal{F}$ необходимо и достаточно, чтобы существовало такое вероятностное распределение $\mu$, сосредоточенное на $[0,1 / 2]$, чTо

$$
\int_{0}^{1} u(x) d G(x)=\int_{0}^{1 / 2}\left[\int_{0}^{1} u(x) d P_{t}(x)\right] d \mu(t)
$$

для любой непрерывной на $[0,1]$ функции и $(x)$.

Док а з а те льст во. Необходимость. В силу свойств элементов класса для всякого $G(x) \in F$ с существует неотрицательная функцция $g(x)$ со свойствами

1) $g(x)=g(1-x), x \in(0,1)$,

2) $g(x)$ не возрастает при $x \in(0,1 / 2]$,

3) $g(x)$ непрерывна справа и слева при $x \in(0,1)$,

4) $G(x)=\int_{0}^{x} g(t) d t+G(+0)$.

Определим меру $\mu(t)$ так, что

$d \mu(0)=2 d G(0), \quad d \mu(1 / 2)=g(1 / 2), \quad d \mu(t)=-2 t d g(t), \quad 1 / 2>t>0$.

Докажем, что определенная так мера является вероятностным распределением, т. е. $\int_{0}^{1 / 2} d \mu(t)=1$.

Имеем

$$
\int_{0}^{1 / 2} d \mu(t)=d \mu(1 / 2)+d \mu(0)-\int_{0}^{1 / 2} 2 t d g(t) .
$$

Интегрируя по частям, получаем

$\int_{0}^{1 / 2} d \mu(t)=g(1 / 2)+2 G(+0)-\left.2 \operatorname{tg}(t)\right|_{0} ^{1 / 2}+2 \int_{+0}^{1 / 2} g(t) d t=2 G(1 / 2)+2 \lim _{t \rightarrow 0} \operatorname{tg}(t)$.

Существование $\lim _{t \rightarrow 0} \operatorname{tg}(t)$ следует из правосторонней непрерывности функции $g(t)$. Пусть $\lim g(t) t=c>0$. Тогда для $0<\varepsilon<c$ существует такое $\delta$, что $\operatorname{tg}(t)>c \stackrel{t \rightarrow 0}{-} \varepsilon$ при $t \in(0, \delta)$, и следовательно,

$$
\int_{0}^{8} g(t) d t>(c-\varepsilon) \int_{0}^{8} \frac{1}{t} d t=\infty,
$$

что противоречит тому, что

$$
\int_{0}^{\delta} g(t) d t=G(\delta)-G(+0)<\infty .
$$

Таким 
образом, $\lim \operatorname{tg}(t)=0$, а $G(1 / 2)=1 / 2$ в силу непрерывности $G(x)$ на $(0,1)$, и окончательно $\int_{u}^{1 / 2} d \mu(t)=1$.

Теперь докажем, что для выбранной меры $\mu$ имеет место равенство (1). Преобразуем правую часть (1):

$$
\begin{gathered}
\int_{0}^{1 / 2}\left(\int_{0}^{1} u(x) d P_{t}(x)\right) d \mu(t)=\int_{t 0}^{1 / 2-0} \int_{0}^{1} u(x) d P_{t}(x)(-2 t) d g(t)+ \\
+d G(0)[u(0)+u(1)]+g(1 / 2) \int_{0}^{1} u(x) d x .
\end{gathered}
$$

Далее, используя вид функции $P_{t}(x)$, получаем

$$
\int_{+0}^{1 / 2-0}\left(\int_{0}^{1} u(x) d P_{t}(x)\right)(-2 t) d g(t)=-\int_{t_{0}}^{1 / 2}\left(\int_{0}^{t}[u(x)+u(1-x)] d x\right) d g(t),
$$

а интегрируя по частям, имеем

$$
\begin{gathered}
-\int_{+0}^{1 / 2-0}\left(\int_{0}^{t}[u(x)+u(1-x)] d x\right) d g(t)=-\left.g(t) \int_{0}^{t}[u(x)+u(1-x)] d x\right|_{+0} ^{1 / 2-0}+ \\
+\int_{0}^{1 / 2+0} g(t)[u(t)+u(1-t)] d t
\end{gathered}
$$

Существование $\lim _{t \rightarrow 0} g(t) \int_{0}^{t}[u(x)+u(1-x)] d x$ следует из правосторонней непрерывности функции $g(t)$. Очевидно,

$$
\left|g(t) \cdot \int_{0}^{t}(u(x)+u(1-x)) d x\right| \leqslant \operatorname{tg}(t) c_{1} \text {, где } c_{1}=\max _{x \leqslant t}|u(x)+u(1-x)|,
$$

a, как доказано ранее, $\lim _{t \rightarrow 0} \operatorname{tg}(t)=0$, и следовательно, $\lim _{t \rightarrow+0} g(t)$. . $\int_{0}^{t}[u(x)+u(1-x)] d x=0 . \quad$ С учетом равенств (3) и (4) получаем

$$
\begin{gathered}
\int_{0}^{1 / 2}\left(\int_{0}^{1} u(x) d P_{t}(x)\right) d \mu(t)= \\
=\int_{0}^{1 / 2} g(t)[u(t)+u(1-t)] d t+d G(0)(u(0)+u \cdot(1))=\int_{0}^{1} u(x) d G(x),
\end{gathered}
$$

что и доказывает необходимость. Достаточность следует из того, что определенная с помощью равенств (2) функция распределения $G(t)$ на основе любого вероятностного распределения $\mu(t)$ принадлежит классу $\mathcal{F}$. Проверка этого факта не представляет трудности с учетом симметричного относительно точки $1 / 2$ доопределения функции $g(t)$ на интервале $(1 / 2,1]$.

Вернемся к доказательству теоремы. Очевидно, что если $\delta \in B(\mathcal{F})$, то $\delta$ является $\mathcal{F}$-допустимой процедурой, так как при выпуклой потере байесовская оценка единственна. Докажем теперь, что если $\delta_{0} \notin B(\mathcal{F})$, то $\delta_{0}$ не является $\mathcal{F}$-допустимой. Рассмотрим функцию $\tilde{r}(\delta, t)=r\left(\delta, P_{t}\right)$, $t \in[0,1 / 2]$. Функцию $\tilde{r}(\delta, t)$ можно интерпретировать как функцию риска при оценивании параметра $t$. В силу теорем А. Вальда $\left[{ }^{3}\right]$ классы допустимых и байесовских оценок параметра $t$ при функции риска $\tilde{r}(\delta, t)$ совпадают. Таким образом, если $U-$ класс всех вероятностных распределений на интервале $[0,1]$, то $\delta^{\prime}$ является допустимой для оценивания параметра $t$ процедурой, если существует $\mu \in U$ такая, что 


$$
\int_{0}^{1 / 2} \tilde{r}\left(\delta^{\prime}, t\right) d \mu(t)=\inf _{\delta} \int_{0}^{1 / 2} \tilde{r}(\delta, t) d \mu(t)
$$

В силу доказанной леммы любому элементу $\mu \in U$ соответствует распределение $F \in \mathcal{F}$ так, что

$$
\int_{0}^{1 / 2} \tilde{r}(\delta, t) d \mu(t)=r(\delta, F) .
$$

Так как по предположению $\delta_{0} \notin \equiv(\widetilde{F})$, то

$$
\inf _{\delta} r(\delta, F)<r\left(\delta_{0}, F\right) \quad \forall F \in \mathcal{F},
$$

откуда в силу равенств (5) и (6) получаем, что $\delta_{0}-$ не байесовская процедура оценивания параметра $t$, а следовательно, и недопустимая. Таким образом, существует процедура $\delta_{1}$ такая, что

$$
\tilde{r}\left(\delta_{1}, t\right) \leqslant \tilde{r}\left(\delta_{0}, t\right) \quad \forall t \in[0,1 / 2],
$$

и ссуществует хотя бы одно $t \in[1 / 2]$, для которого это неравенство строгое. В силу неравенства (7) получаем

$$
\int_{0}^{1 / 2} \tilde{r}\left(\delta_{1}, t\right) d \mu(t) \leqslant \int_{0}^{1 / 2} \tilde{r}\left(\delta_{0}, t\right) d \mu(t) \quad \forall \mu \in U
$$

или

$$
r\left(\delta_{1}, F\right) \leqslant r\left(\delta_{0}, F\right) \quad \forall F \in \mathcal{F},
$$

причем существует хотя бы одно распределение $F \in \mathscr{F}$, для которого последнее неравенство строгое, а это и доказывает, что $\delta_{0}$ не является $\mathcal{F}$-допустимой процедурой.

3 а меч ан ие. Утверждение теоремы сохраняет силу, если вероятностная структура наблюдений и функция риска удовлетворяют допущениям $5.1-5.5$ из [ [ $\left.{ }^{3}\right]$.

Теперь перейдем к определению $\mathcal{F}$-минимаксной процедуры, для чего понадобятся две леммы.

Л ем м а 2. Пусть $\delta_{F}-$ байесовское решение относительно распределения $F \in \mathcal{F}$, T. $e$.

Тогда, если

$$
\inf _{\delta} r(\delta, F)=r\left(\delta_{F}, F\right)
$$

$$
\sup _{G \in \mathscr{F}} r\left(\delta_{F}, G\right)=r\left(\delta_{F}, F\right)
$$

то $\delta_{F}$ является $\mathcal{F}$-минимаксной оценкой.

Д о к а 3 а те льст в о. Напишем очевидные неравенства

$$
\begin{gathered}
\sup _{G \in \mathcal{F}} \inf r(\delta, G)=\sup _{G \in \mathcal{F}} r\left(\delta_{G}, G\right) \geqslant r\left(\delta_{F}, F\right)= \\
=\sup _{G \in \mathcal{F}} r\left(\delta_{F}, G\right) \geqslant \underset{\delta}{\geqslant} \inf _{G \in \mathcal{F}} r(\delta, G) .
\end{gathered}
$$

Крайние члены выписанных отношений удовлетворяют и обратному неравенству, следовательно, везде стоит знак равенства, что и доказывает лемму.

Л ем м а 3. Пусть функция $u(x) \geqslant 0, x \in(0, a)$, где а-фиксированное положительное число, и $и(x)$ - ограниченная неубывающая на $(0, a)$ функция. Определим класс функций $V_{q}(a), q>0$ следующзи образом: 
$V_{q}(a)=\left\{v(x): v(x) \geqslant 0, v(x)\right.$ не возрастает на $\left.(0, a), \int_{0}^{a} v(x) d x=q\right\}$.

Tогда

$$
\sup _{v(x) \in V_{q}(a)} \int_{0}^{a} u(x) v(x) d x=q / a \int_{0}^{a} u(x) d x .
$$

Д ок а $з$ а тельст в о. Пусть $v_{0}(x)=q / a, 0 \leqslant x \leqslant a$. Очевидно, что $v_{0}(x) \in V_{q}(a)$. Для любой функции $v(x) \in V_{q}(a)$, не совпадающей почти всюду на $(0, a)$ с $v_{0}(x)$ существует точка $x_{v} \in(0, a)$ такая, что $v_{0}(x)-v(x)<0$ для $0 \leqslant x \leqslant x_{v}$ и $v_{0}(x)-v(x) \geqslant 0$ для $x_{v}<x \leqslant a$. Это следует из того факта, что $(v(x)-q / a)$ невозрастающая функция, а кроме того, $\underset{v}{\stackrel{a}{f}} v(x) d x=\int_{0}^{a} v_{0}(x) d x$. Таким образсм, имеем

$$
\begin{gathered}
\int_{0}^{a}\left[v_{0}(x)-v(x)\right] u(x) d x= \\
=\int_{0}^{x_{v}}\left[v_{0}(x)-v(x)\right] u(x) d x+\int_{x_{v}}^{a}\left[v_{0}(x)-v(x)\right] u(x) d x \geqslant \\
\geqslant \sup _{0 \leqslant x \leqslant x_{v}}\left(u(x) \cdot \int_{0}^{x_{v}}\left[v_{0}(x)-v(x)\right] d x+\inf _{x_{v} \leqslant x \leqslant a} u(x) \int_{x_{v}}^{a}\left[v_{0}(x)-v(x)\right] d x .\right.
\end{gathered}
$$

В силу неубывания $u(x)$ на $(0, a)$ получаем $\sup _{0 \leqslant x \leqslant x_{v}} u(x)=\inf _{x_{v} \leqslant x \leqslant a} u(x)=$ $=u\left(x_{v}\right)$, и следовательно,

$$
\int_{0}^{a}\left[v_{0}(x)-v(x)\right] u(x) d x \geqslant u\left(x_{v}\right) \int_{0}^{a}\left[v_{0}(x)-v(x)\right] d x=0,
$$

что и доказывает лемму.

Теперь заметим, что $y=\sum_{i=1}^{n} x_{i}$ - достаточная статистика, и следовательно, для оценивания параметра $p$ имеет смысл пользоваться только функциями от $y$, т. е.

$$
r(\delta, G)=\int_{0}^{1} E_{p}(\delta(y)-p)^{2} d G(p)
$$

причем $P(y=k)=C_{n}^{k} p^{k}(1-p)^{n-k}$.

Рассмотрим оценку $\delta_{1 / 2}(y)=\frac{y+1}{n+2}$, которая является байесовской относительно априорного распределения $P_{1 / 2}(p)$. Путем прямых вычислений легко получить

$$
\begin{gathered}
E_{p}\left(\delta_{1 / 2}(y)-p\right)^{2}=\sum_{k=0}^{n}\left(\frac{k+1}{n+2}-p\right)^{2} C_{n}^{k} p^{k}(1-p)^{n-k}= \\
=\frac{p^{2}(4-n)+p(n-4)+1}{(n+2)^{2}} .
\end{gathered}
$$

Пусть $n \geqslant 4$, тогда для $G \in \mathcal{F}$ имеем

$$
r\left(\delta_{1 / 2}, G\right)=2 \int_{0}^{1 / 2} \frac{p^{2}(4-n)+p(n-4)+1}{(n+2)^{2}} g(p) d p+d G(0) \cdot \frac{2}{(n+2)^{2}},
$$


где $g(p)$ и $G(p)$ связаны соотношением $G(p)=\int_{0}^{p} g(t) d t+G(+0)$ (см. теорему), и $\int_{0}^{1 / 2} g(t) d t+G(+0)=1 / 2$. Используя лемму 3 , получаем $r\left(\delta_{1 / 2}, G\right) \leqslant q \frac{n+20}{12(n+2)^{2}}+\left(\frac{1}{2}-q\right) \frac{2}{(n+2)^{2}}=\frac{n-4}{12(n+2)^{2}} q+\frac{2}{(n+2)^{2}}$ где $q=\int_{0}^{1 / 2} g(p) d p \leqslant 1 / 2$. Очевидно, что

$$
\max _{0 \leqslant q \leqslant 1 / 2} \frac{n-4}{12(n+2)^{2}} q+\frac{2}{(n+2)^{2}}=\frac{n+44}{(n+2)^{2}},
$$

и следовательно,

$$
r\left(\delta_{1 / 2} ; G\right) \leqslant \frac{n+44}{(n+2)^{2}} .
$$

В неравенстве (8) в случае $G(p)=P_{1 / 2}(p)$ достигается равенство, откуда по лемме 2 получаем, что $\delta_{1 / 2}(y)-\mathcal{F} \cdot$ минимаксная оценка.

Для случая $n<4$ решением является $\delta_{0}(y)=(y+\sqrt{n / 2}) /(n+\sqrt{n})$, т. е. байесовская оценка, соответствующая априорному распределению с плотностью $C[p(1-p)]^{\sqrt{n / 2}-1}\left[{ }^{3}\right]$.

\section{Л ИТ Е РАТ У РА}

1. Skribinsky, M. SIAM J. Appl. Math., 16, № 1, 134-145 (1968).

2. Skribinsky, M. Ann. Math. Statist., 39, № 2, 492-501 (1968).

3. Вальд А. Статистические решающие функции. - В кн.: Позиционные игры. М., «Наука», 1967, 300-522.

Институт кибернетики

Академии наук Эстонской ССР
Поступила в редакцию 12/VII 1983

\section{OLMAN}

\section{BINOMIAALJAOTUSE PARAMEETRI LUBATAVAD JA BAYESI MINIMAKS- HINNANGUD KITSENDATUD APRIOORSE JAOTUSSEADUSE KORRAL}

On vaadeldud binomiaaljaotuse parameetri Bayesi järgi hindamise ülesannet sōltumatute vaatluste korral tingimusel, et aprioorne jaotusseadus kuulub ühte fikseeritud jaotuse klassi. On tuletatud minimakshinnang ja kirjeldatud lubatavate hinnangute täielik klass.

\section{OLMAN}

\section{ADMISSIBLE AND MINIMAX ESTIMATORS OF THE BINOMIAL PROBABILITY UNDER RESTRICTIONS ON A PRIORI DISTRIBUTION}

The problem of Bayes estimation of the Binomial probability is considered under a condition that an a priori distribution belongs to a fixed class $\mathcal{F}$. Concepts of $\mathcal{F}$-admissibility and $\mathscr{F}$-minimaxity are introduced. The class of all $\mathcal{F}$-admissible estimators, where $F$ consists of distributions $F$ such that $F(p)+F(1-p)=1,0 \leqslant p \leqslant 1$, an $F(p)$ is convex on $[1 / 2,1)$, is described. It is shown that the estimator $\delta$ which is Bayes with respect to the uniform distribution on $\left[\begin{array}{ll}0 & 1\end{array}\right]$, is $\widetilde{\pi}$-minimax. 